\title{
Rectifying full-counting statistics in a spin Seebeck engine
}

\author{
Gaomin Tang, ${ }^{1}$ Xiaobin Chen, ${ }^{1}$ Jie Ren, ${ }^{2, *}$ and Jian Wang ${ }^{1, \dagger}$ \\ ${ }^{1}$ Department of Physics and the Center of Theoretical and Computational Physics, The University of Hong Kong, Hong Kong, China \\ ${ }^{2}$ Center for Phononics and Thermal Energy Science, China-EU Joint Center for Nanophononics, \\ Shanghai Key Laboratory of Special Artificial Microstructure Materials and Technology, \\ School of Physics Science and Engineering, Tongji University, 200092 Shanghai, China
}

(Received 29 May 2017; revised manuscript received 13 October 2017; published 20 February 2018)

\begin{abstract}
In terms of the nonequilibrium Green's function framework, we formulate the full-counting statistics of conjugate thermal spin transport in a spin Seebeck engine, which is made by a metal-ferromagnet insulator interface driven by a temperature bias. We obtain general expressions of scaled cumulant generating functions of both heat and spin currents that hold special fluctuation symmetry relations, and demonstrate intriguing properties, such as rectification and negative differential effects of high-order fluctuations of thermal excited spin current, maximum output spin power, and efficiency. The transport and noise depend on the strongly fluctuating electron density of states at the interface. The results are relevant for designing an efficient spin Seebeck engine and can broaden our view in nonequilibrium thermodynamics and the nonlinear phenomenon in quantum transport systems.
\end{abstract}

DOI: 10.1103/PhysRevB.97.081407

Introduction. Spin caloritronics has emerged as a new field to utilize the excess heat generated in nanodevices to drive spin currents [1]. Of particular interest is the spin Seebeck effect (SSE) in magnetic insulators, which generates pure spin transfer out of the thermal gradient without conducting electron currents, which opens a new direction to harvest heat in the absence of Joule heating [2-9].

The pure spin is carried by magnons (quantized spin waves) that can transfer energy and spin angular momentum. Besides, magnons have a long lifetime so that they can propagate over long distances in a ballistic way without being dissipated in some materials due to low damping [10-12].

Many proposals have been made in exploiting the application of magnons, such as energy harvesters [13], diodes $[8,14]$, transistors [15], and logic devices [16]. In particular, a two-terminal hybrid system involving both metallic and magnonic reservoirs has been proposed [7,17]. In such systems, the propagation of spin waves can be converted to a spin current in the metallic part and vice versa across the junction under a temperature bias. It has been pointed out that a strongly fluctuating electron density of states (DOS) in the metallic materials is helpful to obtain nontrivial rectification and negative differential effects [7,8], which suggests that the quantum dot (QD) structure is a good candidate in achieving these effects. The reduced dimension of QD can also help to increase the figure of merit (FOM) and harvest from the waste heat more efficiently compared to bulk systems when working as a heat engine $[18,19]$.

So far, rectification and negative differential effects have only been studied in the context of the mean values of heat and spin currents. For a nanoscale device, fluctuations and even higher-order cumulants of currents can be significant and

\footnotetext{
*xonics@ tongji.edu.cn

†jianwang@hku.hk
}

play important roles, which could be well described within the framework of full-counting statistics (FCS) [20-27]. FCS of a physical quantity is encoded in its cumulant generating function (CGF), which reveals the higher-order Onsager reciprocity relations [22,28] and the thermodynamic fluctuation symmetry [21,22,29] as well. It would help us in better understanding the nonequilibrium thermodynamics and designing efficient heat harvesters to study the rectification of higher-order cumulants, fluctuation symmetry, and heat engine performances for a two-terminal hybrid system involving both electronic and bosonic reservoirs. An experimental measurement of the full-counting statistics in a mesoscopic conductor has been carried recently [30].

In this Rapid Communication, we investigate the rectification and negative differential effects of FCS in a spin Seebeck engine sandwiched between two reservoirs, one electronic and the other magnonic. We have formulated a framework to obtain the scaled cumulant generating function (SCGF) of both spin and heat currents using the nonequilibrium Green's function (NEGF), which does not require weak couplings to both reservoirs, and treats the interfacial electron-magnon interaction to second order. The fluctuation symmetry is obtained and the conditions for realizing rectification and negative differential effects are discussed. The rectification and negative differential effects of cumulants and heat engine performances with respect to temperature reversal are studied in detail.

Model and theoretical formalism. The system is schematically illustrated in Fig. 1 wherein the left electronic compartment interacts with the right magnonic reservoir. The spin current can be generated out of a temperature bias $\Delta T=$ $T_{L}-T_{R}$. The whole system Hamiltonian has contributions from the electrons, magnons, and the interaction between them, $\hat{H}=\hat{H}_{\mathrm{el}}+\hat{H}_{\mathrm{mag}}+\hat{H}_{\mathrm{em}}$, The electron Hamiltonian $H_{\mathrm{el}}$ is expressed as

$$
\hat{H}_{\mathrm{el}}=\sum_{k \sigma} \epsilon_{k \sigma} a_{k \sigma}^{\dagger} a_{k \sigma} .
$$




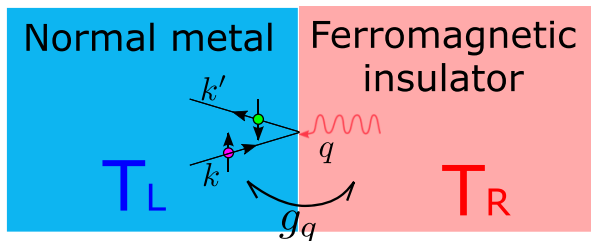

FIG. 1. Schematic illustration of the spin Seebeck engine made by an electron-magnon interface. Heat flowing across the system can induce spin currents due to interfacial electron-magnon coupling.

$\hat{H}_{\text {el }}$ can incorporate the noninteracting scattering region at the interface as well. The electronic spin- $\sigma$ chemical potential is $\mu_{\sigma}$ and their difference is defined as the spin bias with the form $\Delta \mu_{s}=\mu_{\downarrow}-\mu_{\uparrow}$ which could be measured by the inverse spin Hall effect [4]. The Fermi distribution for the spin $\sigma$ is $f_{L \sigma}\left(\epsilon_{k \sigma}\right)=\left\{\exp \left[\beta_{L}\left(\epsilon_{k \sigma}-\mu_{\sigma}\right)\right]+1\right\}^{-1}$, with inverse temperature $\beta_{L}=1 /\left(k_{B} T_{L}\right)$.

The right insulating magnetic compartment can be described by a Heisenberg lattice,

$$
\hat{H}_{\mathrm{mag}}=-J \sum_{\langle i, j\rangle}\left[\frac{1}{2} S_{i+} S_{j-}+\frac{1}{2} S_{i-} S_{j+}+S_{i z} S_{j z}\right],
$$

where $S_{j \pm}$ is the localized raising (lowering) spin operator at site $j, S_{j z}$ is the spin operator in the $z$ direction, and $J$ denotes the exchange coupling strength. Using the Holstein-Primakoff transformation [31,32], $S_{j+}=$ $\sqrt{2 S_{0}-b_{j}^{\dagger} b_{j}} b_{j}, S_{j-}=b_{j}^{\dagger} \sqrt{2 S_{0}-b_{j}^{\dagger} b_{j}}, S_{j z}=S_{0}-b_{j}^{\dagger} b_{j}$ with localized spin length $S_{0}$, the spin operators are mapped into bosonic magnons. At large spin limit $[7,13,19]$, i.e., $2 S_{0} \gg$ $\left\langle b_{j}^{\dagger} b_{j}\right\rangle$, we have the approximation $S_{j}^{-} \approx \sqrt{2 S_{0}} b_{j}^{\dagger}$ and $S_{j}^{+} \approx$ $\sqrt{2 S_{0}} b_{j}$. The right magnetic insulator can be approximated by the free magnon gas $[7,8]$,

$$
\hat{H}_{\mathrm{mag}} \approx \sum_{q} \hbar \omega_{q} b_{q}^{\dagger} b_{q}+\text { const }
$$

after a Fourier transform into the momentum space, where the dispersion of $\omega_{q}$ depends on the material details. The magnonic reservoir obeys the Bose-Einstein distribution $N_{R}(\omega)=1 /\left(e^{\beta_{R} \omega}-1\right)$, where the chemical potential of the magnons is set to zero due to the ferromagnetic insulator phase considered here [33]. The interfacial electron-magnon interaction Hamiltonian bears the form [7,32]

$$
\hat{H}_{\mathrm{em}}=-\sum_{k k^{\prime} q} g_{q}\left[\gamma_{k \uparrow}^{*} \gamma_{k^{\prime} \downarrow} a_{k \uparrow}^{\dagger} a_{k^{\prime} \downarrow} b_{q}^{\dagger}+\text { H.c. }\right] \delta_{\epsilon_{k \uparrow}-\epsilon_{k^{\prime} \downarrow}=\omega_{q}},
$$

with the delta function $\delta_{\epsilon_{k \uparrow}-\epsilon_{k^{\prime} \downarrow}=\omega_{q}}$ ensuring energy and spin angular momentum conservation. The interaction describes a magnon-assisted spin flip by absorbing or emitting magnons with frequency $\omega_{q}$. The effect from the electronic DOS is incorporated in $\gamma_{k \sigma}$. The electron-magnon interaction strength $g_{q}$ is assumed to be weak and will be treated perturbatively in getting SCGF. For simplicity, one can assume $g_{q}$ is energy independent with $g_{q}=g$.

Spin $(s)$ and heat $(h)$ current operators are defined as the change rate of the electronic spin in the left compartment and heat in the right reservoir, respectively, with the forms
$\hat{I}_{s}(t)=-d_{t} \hat{N}_{s}, \hat{I}_{h}(t)=-d_{t} \hat{H}_{R}$, and $d_{t}$ being the total differential with respect to time in the Heisenberg picture. Here, $\hat{N}_{s}=\frac{1}{2} \sum_{k}\left(a_{k \downarrow}^{\dagger} a_{k \downarrow}-a_{k \uparrow}^{\dagger} a_{k \uparrow}\right)$ is the operator of the number of spins in the left compartment and $\hat{H}_{R}=\sum_{q} \hbar \omega_{q} b_{q}^{\dagger} b_{q}$. Using the random phase approximation (RPA), that is, treating the interaction strength $g$ between the electrons and magnon modes perturbatively to the second order [23], we could obtain SCGF for spin and heat current (with counting fields $\lambda_{s}$ and $\lambda_{h}$ ) expressed as (see Supplemental Material [34] for details of the derivation)

$$
\begin{aligned}
\mathcal{G}\left(\lambda_{s}, \lambda_{h}\right)= & -\int \frac{d \omega}{2 \pi} \ln \left\{1-\int \frac{d E}{2 \pi} A\left(E_{-}, E_{+}\right)\right. \\
& \times\left[\left(e^{+i \lambda_{s}+i \hbar \omega \lambda_{h}}-1\right) f_{\downarrow \rightarrow \uparrow}\left(E_{+}, E_{-}\right)\left[N_{R}(\omega)+1\right]\right. \\
& \left.\left.+\left(e^{-i \lambda_{s}-i \hbar \omega \lambda_{h}}-1\right) f_{\uparrow \rightarrow \downarrow}\left(E_{-}, E_{+}\right) N_{R}(\omega)\right]\right\}, \quad(4)
\end{aligned}
$$

with

$$
A\left(E_{-}, E_{+}\right)=\frac{J_{R}(\omega) J_{L \uparrow}\left(E_{-}\right) J_{L \downarrow}\left(E_{+}\right)}{\left|\left[\Sigma_{R}^{r}(\omega)+F^{r}(\omega)\right]\right|^{2}} .
$$

This is our first main result. Here, we have defined $\quad f_{\uparrow \rightarrow \downarrow}\left(E_{-}, E_{+}\right)=f_{L \uparrow}\left(E_{-}\right)\left[1-f_{L \downarrow}\left(E_{+}\right)\right] \quad$ and $f_{\downarrow \rightarrow \uparrow}\left(E_{+}, E_{-}\right)=f_{L \downarrow}\left(E_{+}\right)\left[1-f_{L \uparrow}\left(E_{-}\right)\right] \quad$ with $\quad E_{ \pm}=$ $E \pm \omega / 2 . J_{L \sigma}(E)=2 \pi g \sum_{k}\left|\gamma_{k \sigma}\right|^{2} \delta\left(E-\epsilon_{k \sigma}\right)$ is the spin- $\sigma$ electronic spectral density. The retarded magnonic self-energy due to the dissipation is expressed as $\Sigma_{R}^{r}=-i J_{R}(\omega) / 2$. The expression of the retarded electron-hole propagator is

$$
F^{r}(\omega)=i \int \frac{d \omega_{1}}{2 \pi} \frac{F^{>}\left(\omega_{1}\right)-F^{<}\left(\omega_{1}\right)}{\omega-\omega_{1}+i 0^{+}},
$$

with

$$
\begin{aligned}
& F^{<}(\omega)=-i \int \frac{d E}{2 \pi}\left[J_{\uparrow}\left(E_{-}\right) J_{\downarrow}\left(E_{+}\right) f_{\downarrow \rightarrow \uparrow}\left(E_{+}, E_{-}\right)\right], \\
& F^{>}(\omega)=-i \int \frac{d E}{2 \pi}\left[J_{\uparrow}\left(E_{-}\right) J_{\downarrow}\left(E_{+}\right) f_{\uparrow \rightarrow \downarrow}\left(E_{-}, E_{+}\right)\right] .
\end{aligned}
$$

The physical picture of SCGF is clear. The first contribution of Eq. (4) describes that the spin-down electrons with energy $E+\hbar \omega / 2$ flip to the spin-up states with energy $E-\hbar \omega / 2$ by emitting a magnon with energy $\hbar \omega$ into the magnonic reservoir. The second one describes a reverse process where the spin-up electrons with energy $E-\hbar \omega / 2$ flip to the spin-down states with energy $E+\hbar \omega / 2$ via absorbing a magnon carrying energy $\hbar \omega$.

Applying the identity $f_{\uparrow \rightarrow \downarrow}\left(E_{-}, E_{+}\right)=e^{\beta_{L}\left(\omega-\Delta \mu_{s}\right)} f_{\downarrow \rightarrow \uparrow}$ $\left(E_{+}, E_{-}\right)$in Eq. (4), we can obtain the following fluctuation symmetry relation,

$$
\mathcal{G}\left(\lambda_{s}, \lambda_{h}\right)=\mathcal{G}\left(-\lambda_{s}-i \beta_{L} \Delta \mu_{s},-\lambda_{h}+i\left(\beta_{L}-\beta_{R}\right)\right) .
$$

The fluctuation relation is generalized in the fermion-boson hybrid junction. This is our second main result. The spin current in the electronic reservoir and heat current in the magnonic reservoir could be derived by taking the first-order derivative of $\mathcal{G}(\{\lambda\})$ with respect to $\lambda_{s}$ and $\lambda_{h}$, respectively, at $\lambda_{s}=\lambda_{h}=0$, that is, $I_{s / q}=\partial \mathcal{G}(\{\lambda\}) /\left.\partial\left(i \lambda_{s / q}\right)\right|_{\lambda_{s}=\lambda_{h}=0}$. 
Then,

$$
\begin{aligned}
I_{s(h)}= & \int \frac{d \omega}{2 \pi} \int \frac{d E}{2 \pi}\left\{( \hbar \omega ) ^ { v } A ( E _ { - } , E _ { + } ) \left[f_{\downarrow \rightarrow \uparrow}\left(E_{+}, E-\right)\right.\right. \\
& \left.\left.\times\left(N_{R}(\omega)+1\right)-f_{\uparrow \rightarrow \downarrow}\left(E_{-}, E_{+}\right) N_{R}(\omega)\right]\right\},
\end{aligned}
$$

where $v=0$ for spin current $I_{s}$, and $v=1$ for heat current $I_{h}$.

If $J_{L \sigma}(E)$ were flat near the Fermi energy, $A\left(E_{-}, E_{+}\right)$ inside the integral in Eq. (4) would be treated as a constant. By applying the equality $\int \frac{d E}{2 \pi} f_{\downarrow \rightarrow \uparrow}\left(E_{+}, E_{-}\right)=(\hbar \omega-$ $\left.\Delta \mu_{s}\right) N_{L}\left(\hbar \omega-\Delta \mu_{s}\right)$ and assuming $\Delta \mu_{s} \rightarrow 0$, SCGF would be the same by reversing the signs of counting fields and exchanging the temperatures of two reservoirs, $\mathcal{G}\left(\lambda_{s}, \lambda_{h}\right)=$ $\mathcal{G}\left(-\lambda_{s},-\lambda_{h} ; T_{L} \leftrightarrow T_{R}\right)$. This implies that the cumulants of the currents would be symmetric by reversing the temperature gradient $\Delta T$ for the system with a constant electron DOS. This is usually satisfied in the pure fermionic system or bosonic system, but is broken when DOS $J_{L \sigma}(E)$ is strongly fluctuating [see Eq. (10) for small $\Gamma_{\sigma}$ ] in the electron-magnon hybrid system discussed here. Therefore, a strongly fluctuating electron DOS is essential to have asymmetric behavior with respect to reversing the temperature gradient and rectifying FCS and the heat engine performances of the spin Seebeck engine. This is our third main result.

Numerical results. In order to get a fluctuating electron DOS, one can, for example, insert a QD between the electronic and magnonic reservoir. The Hamiltonian of the electronic part is then expressed as

$$
\hat{H}_{\mathrm{el}}=\sum_{\sigma} \epsilon_{\sigma} d_{\sigma}^{\dagger} d_{\sigma}+\sum_{k \sigma}\left[\epsilon_{k \sigma} c_{k \sigma}^{\dagger} c_{k \sigma}+\left(t_{k \sigma} c_{k \sigma}^{\dagger} d_{\sigma}+\text { H.c. }\right)\right] \text {, }
$$

with the first term describing QD, the second term the electronic reservoir, and the rest for their coupling. $\epsilon_{\sigma}$ are the two energy levels inside QD. We consider a large quantum dot so that the Coulomb interaction effect can be neglected. $\epsilon_{k \sigma}$ and $t_{k \sigma}$ are the energy level of state $k \sigma$ in the electronic reservoir and its coupling strength with the spin $\sigma$ in QD. The electron Hamiltonian $\hat{H}_{\mathrm{el}}$ with QD can be diagonalized and rewritten in the form of Eq. (1) through the linear transformation [7,35]

$$
d_{\sigma}=\sum_{k} \gamma_{k \sigma} a_{k \sigma}, \quad c_{k \sigma}=\sum_{k^{\prime}} \eta_{k k^{\prime} \sigma} a_{k^{\prime} \sigma},
$$

where the dimensionless coefficients are

$$
\gamma_{k \sigma}=\frac{t_{k \sigma}}{\epsilon_{k \sigma}-\epsilon_{\sigma}-\Sigma_{k \sigma}}, \quad \eta_{k k^{\prime} \sigma}=\delta_{k k^{\prime}}-\frac{t_{k \sigma} \gamma_{k^{\prime} \sigma}}{\epsilon_{k \sigma}-\epsilon_{k^{\prime} \sigma}+i 0^{+}},
$$

with the self-energy function $\Sigma_{k \sigma}=\sum_{k^{\prime}} t_{k^{\prime} \sigma}^{2} /\left(\epsilon_{k}-\epsilon_{k^{\prime}}+\right.$ $\left.i 0^{+}\right)$. The electronic spectral densities in the left compartment (electronic reservoir plus QD) are Lorentzian shaped with the form [35]

$$
J_{L \sigma}(E)=g \Gamma_{\sigma} /\left[\left(E-\epsilon_{\sigma}\right)^{2}+\Gamma_{\sigma}^{2} / 4\right],
$$

where the electronic coupling strength $\Gamma_{\sigma}=$ $2 \pi \sum_{k}\left|t_{k \sigma}\right|^{2} \delta\left(E-\epsilon_{k \sigma}\right)$ is assumed energy independent. Without loss of generality, the magnonic reservoir spectral function is considered to be Ohmic and is expressed as $J_{R}(\omega)=\pi \alpha \omega e^{-\omega / \omega_{c}}$, where $\alpha$ describes the strength of dissipation into the magnonic reservoir and $\omega_{c}$ is the cutoff frequency $[34,36]$.

During the numerical calculation, the coupling strengths for the spin-up and spin-down electrons between the electronic
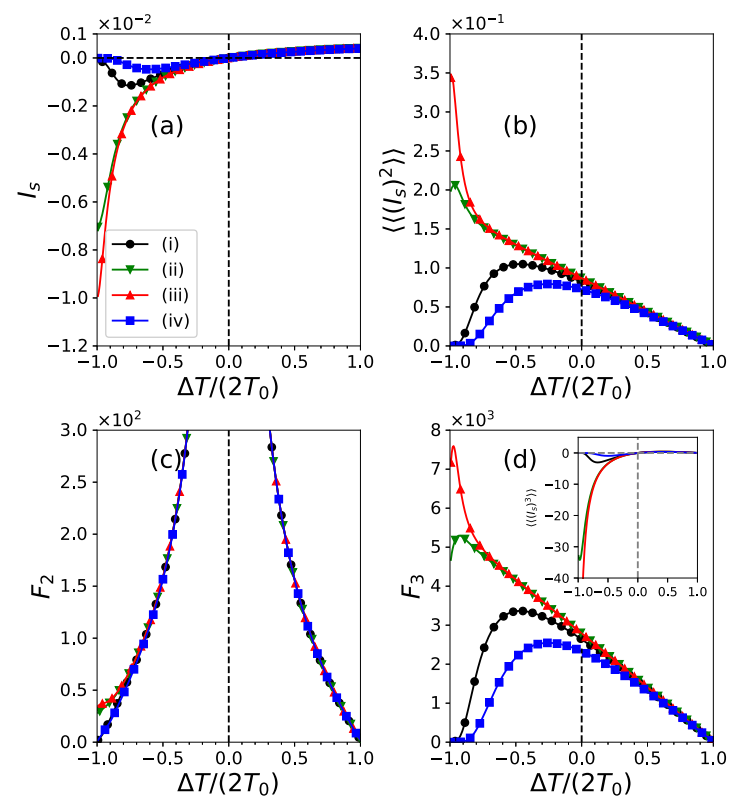

FIG. 2. (a) Spin current $I_{s}$, (b) spin current noise $\left\langle\left\langle\left(I_{s}\right)^{2}\right\rangle\right\rangle$, (c) Fano factor $F_{2}$, and (d) the renormalized third-order spin current cumulant $F_{3}=\left\langle\left\langle\left(I_{s}\right)^{3}\right\rangle\right\rangle /\left\langle I_{s}\right\rangle$ as a function of $\Delta T /\left(2 T_{0}\right)$ by varying $\epsilon_{\sigma}$ : (i) $\epsilon_{\downarrow}=15 \mathrm{meV}, \epsilon_{\uparrow}=10 \mathrm{meV}$; (ii) $\epsilon_{\downarrow}=5 \mathrm{meV}, \epsilon_{\uparrow}=0$; (iii) $\epsilon_{\downarrow}=3 \mathrm{meV}, \epsilon_{\uparrow}=-2 \mathrm{meV}$; (iv) $\epsilon_{\downarrow}=-20 \mathrm{meV}, \epsilon_{\uparrow}=-25 \mathrm{meV}$. The third-order spin current cumulant $\left\langle\left\langle\left(I_{s}\right)^{3}\right\rangle\right\rangle$ is plotted as an inset in (d). $\Gamma=6 \mathrm{meV}$.

reservoir and QD are assumed to be energy independent and equal, i.e., $\Gamma_{\uparrow}=\Gamma_{\downarrow}=\Gamma$. The energy level of spin down is set to be larger than that of spin up in the QD, $\epsilon_{\downarrow}>\epsilon_{\uparrow}$. The dimensionless dissipation strength and the cutoff frequency of the magnonic reservoir are chosen as $\alpha=0.2$, and $\omega_{c}=$ $80 \mathrm{meV}$, respectively. The temperatures of the reservoirs are set as $T_{L, R}=T_{0} \pm \Delta T / 2$ with $T_{0}=300 \mathrm{~K}$, so that when the normalized temperature gradient $\Delta T /\left(2 T_{0}\right)<0$, the left lead is cooler. We denote the average of spin-up and spin-down chemical potentials as $\mu_{0}=\left(\mu_{\uparrow}+\mu_{\downarrow}\right) / 2$. The spin currents are displayed in atomic units.

In Fig. 2, we plot the spin current $I_{s}$ [Fig. 2(a)], spin current noise $\left\langle\left\langle\left(I_{s}\right)^{2}\right\rangle\right\rangle$ [Fig. 2(b)], Fano factor $F_{2}=\left\langle\left\langle\left(I_{s}\right)^{2}\right\rangle\right\rangle / I_{s}$ [Fig. 2(c)], and the renormalized third-order spin current cumulant $F_{3}=\left\langle\left\langle\left(I_{s}\right)^{3}\right\rangle\right\rangle /\left\langle I_{s}\right\rangle$ [Fig. 2(d)] as a function of the normalized temperature gradient $\Delta T /\left(2 T_{0}\right)$ by varying $\epsilon_{\sigma}$. The third-order spin current cumulant $\left\langle\left\langle\left(I_{s}\right)^{3}\right\rangle\right\rangle$ is plotted as an inset in Fig. 2(d). The spin-up and spin-down chemical potentials are both set to zero, i.e., $\mu_{\uparrow}=\mu_{\downarrow}=0$. The rectification effect where the spin currents are asymmetric with respect to temperature gradient reversal can be clearly identified in Fig. 2. In the cases where the two levels of QD are either both above or below the chemical potential $\left[\epsilon_{\downarrow}>\epsilon_{\uparrow}>0\right.$ in case (i) or $0>\epsilon_{\downarrow}>\epsilon_{\uparrow}$ in case (iv) in Fig. 2], we could observe the negative differential SSE that the spin currents decrease or even vanish with increasing temperature gradient $\Delta T$. This could be understood as follows: When the right reservoir is hotter, i.e., $\Delta T<0$, the process where the spin-up electron absorbs energy to flip to the spin-down state dominates. Near the linear response regime, increasing the magnitude of the temperature gradient which corresponds to decreasing $T_{L}$ leads to an increased heat 

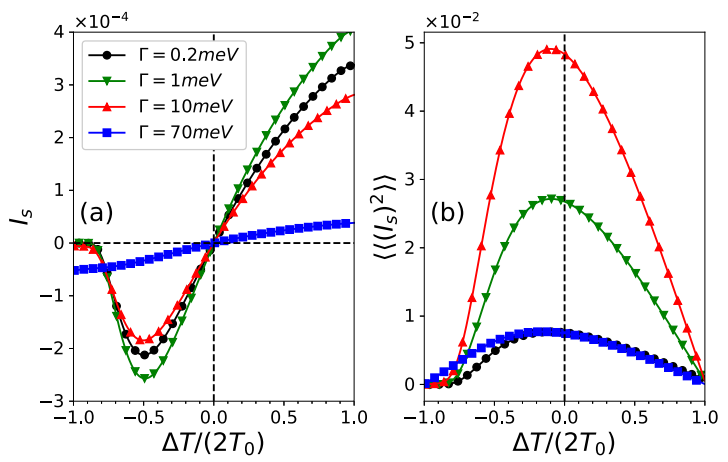

FIG. 3. (a) Spin current, and (b) spin current noise as a function of $\Delta T /\left(2 T_{0}\right)$, by varying electronic coupling strength $\Gamma$, with $\epsilon_{\uparrow}=$ $30 \mathrm{meV}$ and $\epsilon_{\downarrow}=35 \mathrm{meV}$.

current to assist the spin-flip process. When the two levels are both above (below) the chemical potential, the electrons on these levels can be depleted (occupied) by further decreasing $T_{L}$. Compared to the increasing $N_{R}(\omega), f_{\uparrow \rightarrow \downarrow}\left(E_{-}, E_{+}\right)=$ $f_{L \uparrow}\left(E_{-}\right)\left[1-f_{L \downarrow}\left(E_{+}\right)\right]$is severely suppressed and so is the flipping process from the spin-up to the spin-down state [7]. Thus, the essential ingredient to get negative differential SSE is that the two levels are on one side of the chemical potential. We can also observe from Fig. 2(b) that spin current noise is asymmetric with respect to the temperature gradient which signifies rectification as well. If $J_{L \sigma}(E)$ is flat near $\epsilon_{\sigma}$, spin current noise is an even function of $\Delta T$, which is not satisfied in Fig. 2(b). For cases (ii) and (iii), noise is monotonic, while for cases (i) and (iv), a decrease in noise with increasing the magnitude of $\Delta T\left[\Delta T /\left(2 T_{0}\right)<-0.5\right]$ is due to the severely suppressed number of electrons involved in the spin flip. One can observe that the Fano factors shown in Fig. 2(c) are relatively symmetric and almost the same for all cases, except in the region $\Delta T /\left(2 T_{0}\right) \rightarrow-1$. The asymmetries of $\left\langle\left\langle\left(I_{S}\right)^{3}\right\rangle\right\rangle$ and $F_{3}$ with respect to the temperature gradient reversal can be clearly identified. We can even observe the negative differential effect of $\left\langle\left\langle\left(I_{s}\right)^{3}\right\rangle\right\rangle$ in cases (ii) and (iii), which is absent for $\left\langle I_{s}\right\rangle$. Due to the level broadening in the electronic reservoir, electrons with energy levels above (below) the chemical potential can be partially depleted (occupied) by decreasing $T_{L}$. This demonstrates that the third-order cumulant is more sensitive than the spin current in the negative differential effect.

The spin currents and spin current noise versus $\Delta T /\left(2 T_{0}\right)$ by varying the electronic coupling strength $\Gamma$ with $\epsilon_{\uparrow}=30 \mathrm{meV}$ and $\epsilon_{\downarrow}=35 \mathrm{meV}$ are shown in Figs. 3(a) and 3(b), respectively. With increasing $\Gamma$, the spin current increases first and then decreases. When $\Gamma$ is very small, $\Gamma \approx 0.2 \mathrm{meV}$, the electronic spectral density is quite small so that the spin flip is limited by $\Gamma$ and the spin current will increase with increasing $\Gamma$. However, when $\Gamma$ is large, increasing $\Gamma$ reduces the spin current because of the level broadening. The negative differential SSE disappears once $\Gamma$ is too large, since the electron DOS becomes flatter near $\epsilon_{\sigma}$ with increasing $\Gamma$. Spin current noise is getting symmetric with increasing $\Gamma$ due to electron DOS flattening, and this holds as well for the case of $\epsilon_{\downarrow}>0>\epsilon_{\uparrow}$, which is not shown here.

We now discuss the device working as a thermoelectric engine with the output spin power $P=\left|I_{s} \Delta \mu_{s}\right|$. Since the spin

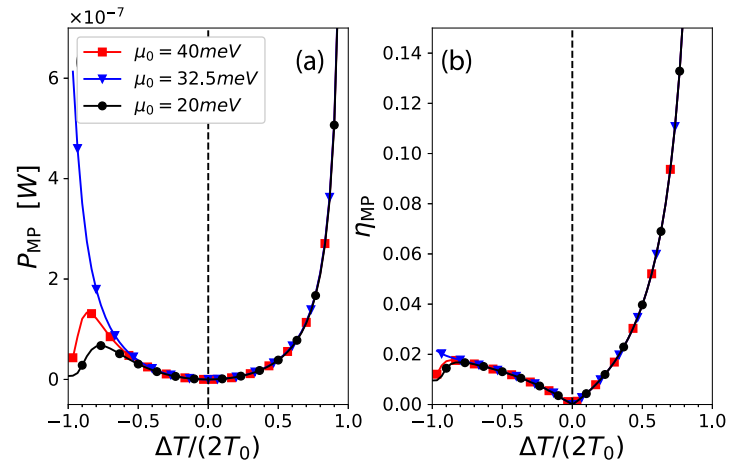

FIG. 4. (a) Maximum output spin power $P_{\mathrm{MP}}$, and (b) efficiency $\eta_{\mathrm{MP}}$ at maximum power as a function of $\Delta T /\left(2 T_{0}\right)$ by varying $\mu_{0}$ with $\epsilon_{\uparrow}=30 \mathrm{meV}, \epsilon_{\downarrow}=35 \mathrm{meV}$, and $\Gamma=4 \mathrm{meV}$.

current does not generate heat, the efficiency should be defined as $\eta=P /\left|I_{h}\right|$. When $\Delta T=T_{L}-T_{R}<0$, heat current flows from the right reservoir to drive the spin current, and the heat current calculated from Eq. (8) is negative. One should note that the spin bias which is defined as $\Delta \mu_{s}=\mu_{\downarrow}-\mu_{\uparrow}$ should be chosen as positive $\Delta \mu_{s}>0$ under $\Delta T<0$, otherwise the device works as an unphysical dud engine where the output spin power heats both reservoirs. In the case of $\Delta T>0$, i.e., $T_{L}>T_{R}$, the spin bias $\Delta \mu_{s}<0$.

For a heat engine, maximum output power and maximum efficiency cannot be satisfied at the same time. One usually cares about the efficiency $\eta_{\text {MP }}$ when the system has a maximum power $P_{\mathrm{MP}}$ or an output power under maximum efficiency at a given $\Delta T$ [37]. In Fig. 4, we plot the maximum output spin power $P_{\mathrm{MP}}$, and efficiency $\eta_{\mathrm{MP}}$ at maximum power as a function of $\Delta T /\left(2 T_{0}\right)$ by varying the average chemical potential $\mu_{0}$. For the case of $\mu_{0}=32.5 \mathrm{meV}$, where $\mu_{0}$ is between $\epsilon_{\uparrow}$ and $\epsilon_{\downarrow}, P_{\mathrm{MP}}$ would be relatively symmetric due to the different asymmetries of spin current and spin bias at maximum power [34]. $\eta_{\text {MP }}$ for all three cases is asymmetric with respect to the temperature reversal due to the rectification effect of the heat current, which is the denominator in calculating efficiency. When $\mu_{0}$ is above or below both levels $\epsilon_{\sigma}$ of the QD, we can observe the negative differential effect of both $P_{\mathrm{MP}}$ and $\eta_{\mathrm{MP}}$, which is also due to the severely decreased electron number near $\epsilon_{\sigma}$ with decreasing $T_{L}$.

Conclusion. In this Rapid Communication, we have uncovered the rectification and negative differential effects of FCS and heat engine performances in an electron-magnon diode under a temperature gradient. The SCGF of spin and heat flow of the system were obtained in the framework of NEGF. Spin and heat current expressions were derived and fluctuation symmetry was verified from the SCGF. We verified that the strongly fluctuating electron DOS induced by the QD is crucial to get these intriguing effects. In principle, multiple QDs can be sandwiched between metallic and magnonic reservoirs to form multiple transport channels in parallel to enhance transport. The hybrid two-terminal setup involving both electronic and bosonic reservoirs exhibits a perfect testing ground for nontrivial phenomena in nonequilibrium thermodynamics.

Acknowledgments. G.T., X.C., and J.W. are financially supported by the General Research Fund (Grant No. 17311116), the University Grant Council (Contract No. AoE/P-04/08) 
of the Government of HKSAR, and NSF-China (Grant No. 11374246). J.R. acknowledges support from the NSFC with
Grant No. 11775159, the National Youth 1000 Talents Program in China, and the Startup Grant at Tongji University.
[1] G. E. W. Bauer, E. Saitoh, and B. J. van Wees, Nat. Mater. 11, 391 (2012).

[2] K. Uchida, S. Takahashi, K. Harii, J. Ieda, W. Koshibae, K. Ando, S. Maekawa, and E. Saitoh, Nature (London) 455, 778 (2008).

[3] Y. Kajiwara, K. Harii, S. Takahashi, J. Ohe, K. Uchida, M. Mizuguchi, H. Umezawa, H. Kawai, K. Ando, K. Takanashi, S. Maekawa, and E. Saitoh, Nature (London) 464, 262 (2010).

[4] K. Uchida K. Uchida, J. Xiao, H. Adachi, J. Ohe, S. Takahashi, J. Ieda, T. Ota, Y. Kajiwara, H. Umezawa, H. Kawai, G. E. W. Bauer, S. Maekawa, and E. Saitoh, Nat. Mater. 9, 894 (2010).

[5] A. Slachter, F. L. Bakker, J.-P. Adam, and B. J. van Wees, Nat. Phys. 6, 879 (2010).

[6] H. Adachi, K. Uchida, E. Saitoh, and S. Maekawa, Rep. Prog. Phys. 76, 036501 (2013).

[7] J. Ren, Phys. Rev. B 88, 220406(R) (2013).

[8] J. Ren and J.-X. Zhu, Phys. Rev. B 88, 094427 (2013).

[9] F. Ronetti, L. Vannucci, G. Dolcetto, M. Carrega, and M. Sassetti, Phys. Rev. B 93, 165414 (2016).

[10] J. Xiao, G. E. W. Bauer, K.-C. Uchida, E. Saitoh, and S. Maekawa, Phys. Rev. B 81, 214418 (2010).

[11] U. Ritzmann, D. Hinzke, and U. Nowak, Phys. Rev. B 89, 024409 (2014).

[12] J. Barker and G. E. W. Bauer, Phys. Rev. Lett. 117, 217201 (2016).

[13] B. Sothmann and M. Büttiker, Europhys. Lett. 99, 27001 (2012).

[14] S. Borlenghi, S. Lepri, L. Bergqvist, and A. Delin, Phys. Rev. B 89, 054428 (2014).

[15] A. V. Chumak, A. A. Serga, and B. Hillebrands, Nat. Commun. 5, 4700 (2014).

[16] B. Lenk, H. Ulrichs, F. Garbs, and M. Münzenberg, Phys. Rep. 507, 107 (2011).

[17] Ł. Karwacki, P. Trocha, and J. Barnaś, Phys. Rev. B 92, 235449 (2015).

[18] M. Esposito, R. Kawai, K. Lindenberg, and C. Van den Broeck, Phys. Rev. E 81, 041106 (2010); N. Nakpathomkun, H. Q. Xu, and H. Linke, Phys. Rev. B 82, 235428 (2010).

[19] B. Sothmann, R. Sánchez, and A. N. Jordan, Nanotechnology 26, 032001 (2015).

[20] L. S. Levitov, H.-W. Lee, and G. B. Lesovik, J. Math. Phys. 37, 4845 (1996); L. S. Levitov, in Quantum Noise in Mesoscopic Physics, edited by Yu. V. Nazarov, NATO Science Series II Vol. 97 (Kluwer, Dordrecht, 2003).
[21] M. Esposito, U. Harbola, and S. Mukamel, Rev. Mod. Phys. 81, 1665 (2009).

[22] M. Campisi, P. Hänggi, and P. Talkner, Rev. Mod. Phys. 83, 771 (2011).

[23] A. Kamenev, Field Theory of Non-Equilibrium Systems (Cambridge University Press, Cambridge, UK, 2011).

[24] G.-M. Tang and J. Wang, Phys. Rev. B 90, 195422 (2014); Z. Yu, G.-M. Tang, and J. Wang, ibid. 93, 195419 (2016); G. Tang, Z. Yu, and J. Wang, New J. Phys. 19, 083007 (2017).

[25] C. Flindt, T. Novotný, A. Braggio, M. Sassetti, and A. P. Jauho, Phys. Rev. Lett. 100, 150601 (2008); C. Flindt, T. Novotný, A. Braggio, and A. P. Jauho, Phys. Rev. B 82, 155407 (2010); V. F. Maisi, D. Kambly, C. Flindt, and J. P. Pekola, Phys. Rev. Lett. 112, 036801 (2014).

[26] B. K. Agarwalla, B. Li, and J.-S. Wang, Phys. Rev. E 85, 051142 (2012); B. K. Agarwalla, H. Li, B. Li, and J.-S. Wang, ibid. 89, 052101 (2014).

[27] R. S. Souto, A. Martín-Rodero, and A. L. Yeyati, Phys. Rev. Lett. 117, 267701 (2016); Phys. Rev. B 96, 165444 (2017).

[28] D. Andrieux and P. Gaspard, J. Chem. Phys. 121, 6167 (2004).

[29] D. Andrieux and P. Gaspard, J. Stat. Mech. (2006) P01001.

[30] S. Singh, J. T. Peltonen, I. M. Khaymovich, J. V. Koski, C. Flindt, and J. P. Pekola, Phys. Rev. B 94, 241407(R) (2016); D. Dasenbrook and C. Flindt, Phys. Rev. Lett. 117, 146801 (2016).

[31] T. Holstein and H. Primakoff, Phys. Rev. 58, 1098 (1940).

[32] G. D. Mahan, Many-Particle Physics, 3rd ed. (Kluwer Academic, Dordrecht, 2000).

[33] K. A. van Hoogdalem, M. Albert, P. Simon, and D. Loss, Phys. Rev. Lett. 113, 037201 (2014).

[34] See Supplemental Material at http://link.aps.org/supplemental/ 10.1103/PhysRevB.97.081407 for the derivation of scaled cumulant generating function using nonequilibrium Green's function.

[35] B. K. Agarwalla, J.-H. Jiang, and D. Segal, Phys. Rev. B 92, 245418 (2015); L. Simine and D. Segal, Phys. Chem. Chem. Phys. 14, 13820 (2012).

[36] U. Weiss, Quantum Dissipative Systems (World Scientific, Singapore, 2012).

[37] M. Esposito, K. Lindenberg, and C. Van den Broeck, Phys. Rev. Lett. 102, 130602 (2009); M. Esposito, N. Kumar, K. Lindenberg, and C. Van den Broeck, Phys. Rev. E 85, 031117 (2012); K. Proesmans, B. Cleuren, and C. Van den Broeck, Phys. Rev. Lett. 116, 220601 (2016). 